\title{
Effect of red blood cells on platelet activation and thrombus formation in tortuous arterioles
}

\author{
Jennifer K. W. Chesnutt ${ }^{1,2}$ and Hai-Chao Han ${ }^{1,3} *$ \\ ' Cardiovascular Biomechanics Laboratory, Department of Mechanical Engineering, The University of Texas at San Antonio, San Antonio, TX, USA \\ 2 Department of Pathology, University of Texas Health Science Center at San Antonio, San Antonio, TX, USA \\ ${ }^{3}$ Biomedical Engineering Program, UTSA-UTHSCSA, San Antonio, TX, USA
}

\section{Edited by:}

Tim Harrigan, Johns Hopkins

University Applied Physics

Laboratory, USA

\section{Reviewed by:}

Zhi-Yong Li, Southeast University, China

Seungik Baek, Michigan State

University, USA

Haiyan Luo, Cooper Union, USA

Yang Liu, The Hong Kong Polytechnic

University, Hong Kong

\section{*Correspondence:}

Hai-Chao Han, Department of Mechanical Engineering, The

University of Texas at San Antonio,

One UTSA Circle, San Antonio, TX

78249, USA

e-mail:hchan@utsa.edu
Thrombosis is a major contributor to cardiovascular disease, which can lead to myocardial infarction and stroke. Thrombosis may form in tortuous microvessels, which are often seen throughout the human body, but the microscale mechanisms and processes are not well understood. In straight vessels, the presence of red blood cells (RBCs) is known to push platelets toward walls, which may affect platelet aggregation and thrombus formation. However in tortuous vessels, the effects of RBC interactions with platelets in thrombosis are largely unknown. Accordingly, the objective of this work was to determine the physical effects of RBCs, platelet size, and vessel tortuosity on platelet activation and thrombus formation in tortuous arterioles. A discrete element computational model was used to simulate the transport, collision, adhesion, aggregation, and shear-induced platelet activation of hundreds of individual platelets and RBCs in thrombus formation in tortuous arterioles. Results showed that high shear stress near the inner sides of curved arteriole walls activated platelets to initiate thrombosis. RBCs initially promoted platelet activation, but then collisions of RBCs with mural thrombi reduced the amount of mural thrombus and the size of emboli. In the absence of RBCs, mural thrombus mass was smaller in a highly tortuous arteriole compared to a less tortuous arteriole. In the presence of RBCs however, mural thrombus mass was larger in the highly tortuous arteriole compared to the less tortuous arteriole. As well, smaller platelet size yielded less mural thrombus mass and smaller emboli, either with or without RBCs. This study shed light on microscopic interactions of RBCs and platelets in tortuous microvessels, which have implications in various pathologies associated with thrombosis and bleeding.

Keywords: erythrocytes, shear-induced platelet activation, tortuosity, thrombosis, microvessels, mean platelet volume, computational simulation, discrete element model

\section{INTRODUCTION}

Tortuous microvessels are found throughout the human body, such as coronary vasculature ( Hutchins et al., 1978), and cerebral (Spangler et al., 1994; Brown et al., 2002), retinal (Sasongko et al., 2012), and conjunctival (Owen et al., 2008) arteries. Microvascular tortuosity alters blood flow to increase fluid shear stress that can activate platelets and induce thrombosis, even in the absence of vessel injury and hypercoagulability. For example, an in vivo study showed that thrombus formed due to high shear stress in rat venules that were made curved from an originally straight shape, but did not form in straight venules (Liu et al., 2008). As well, microvascular thrombi have been observed in humans in clinical, experimental, and autopsy settings (Gando, 2010). Consequently, the study of platelet activation and thrombus formation in tortuous microvessels is of clinical importance.

In straight vessels in vivo, large numbers of red blood cells (RBCs) flow in the vessel pushing platelets to the wall, known as platelet margination as demonstrated in vitro (Aarts et al., 1988). Thus, platelets are highly concentrated near the lumen walls. Responses of platelet margination and platelet adhesion rates to RBC collisions, hematocrit, and platelet size have been analyzed using mathematical models (Tokarev et al., 2011a,b). Also, the microscale processes of RBC and platelet interactions have been analyzed using computational simulations tracking individual cells. These simulations have been used effectively to examine the effects of RBC collisions, RBC aggregation, RBC deformability, RBC cytoplasm viscosity, platelet size and shape, platelet adhesion forces, and channel size on platelet margination, shear forces on platelets, and the process of thrombus formation (Miyazaki and Yamaguchi, 2003; AlMomani et al., 2008; Mori et al., 2008a; Chesnutt and Marshall, 2009a; Kamada et al., 2012; Reasor et al., 2012). Though these previous models have elucidated effects of RBCs in straight vessels and channels, effects of RBCs in tortuous vessels have not been addressed. In non-cylindrical vessels, a few in vitro studies with blood flow through sudden expansions showed that hematocrit affected spatial concentrations of RBCs and plateletsized particles (Zhao et al., 2008) and affected platelet adhesion (Karino and Goldsmith, 1984). Hence, RBCs also likely play a role in thrombosis in tortuous vessels.

Most computational simulations that modeled thrombus formation by tracking individual platelets were performed in straight tubes or channels without RBCs (Miyazaki and Yamaguchi, 2003; 
Pivkin et al., 2006; Filipovic et al., 2008b; Fogelson and Guy, 2008; Mori et al., 2008b; Kamada et al., 2010), and very few included RBCs (Mori et al., 2008a; Xu et al., 2009; Kamada et al., 2012). Few simulations have been accomplished in non-cylindrical geometries (e.g., stenosis or tubular expansion), and these studies were without RBCs (Filipovic et al., 2008a; Kamada et al., 2011). In most of these models, platelets were activated due to an injured segment of the vessel wall. The effects of platelet activation by high shear stress were not studied.

Therefore, we have previously simulated the microscale processes of thrombus formation in tortuous arterioles and venules based on shear-induced activation of individual platelets (Chesnutt and Han, 2011, 2013). However, these studies did not include RBCs in the simulations.

Another physical factor that is present in conditions associated with many thrombotic and bleeding complications is platelet size, which is measured clinically as mean platelet volume (MPV). For normal healthy human subjects, an increase in MPV was shown to increase platelet aggregation in platelet rich plasma (Karpatkin, 1978). Elevated MPV is observed in pathological conditions, including diabetes (Papanas et al., 2004), hypertrophic cardiomyopathy (Cambronero et al., 2009), acute myocardial infarction (Chu et al., 2010), restenosis following coronary angioplasty (Chu et al., 2010), pulmonary hypertension (Guvenc et al., 2012), and giant platelet disorders (Mhawech and Saleem, 2000). MPV also decreases under other pathological conditions, such as reactive systemic amyloid A amyloidosis (Erdem et al., 2012) and WiskottAldrich syndrome (Ochs et al., 1980). Hence, it is also of interest to determine the effects of platelet size in the presence of RBCs.

Consequently, the objective of this work was to determine the physical effects of RBCs, MPV, and vessel tortuosity on shearinduced platelet activation and thrombus formation in tortuous arterioles. These results would help to develop new treatment strategies and diagnostic tests for the risk of cardiovascular disease.

\section{MATERIALS AND METHODS}

The transport, collision, activation, adhesion, and aggregation processes of hundreds of individual RBCs and platelets of different MPV were numerically simulated in tortuous arterioles. The computational simulation conditions are described first, followed by brief descriptions of the employed discrete element model and shear-induced platelet activation model.

\section{COMPUTATIONAL SIMULATION CONDITIONS}

Segments of tortuous arterioles, each having diameter $D=25 \mu \mathrm{m}$, were modeled as two-dimensional (2D) channels in the shapes of cosine curves with two periods. Walls near the bends (locations with highest curvature) were designated as either inner or outer walls (Figure 1). A tortuosity index $T$ was defined as the ratio of amplitude $A$ to wavelength $\lambda$ of the cosine curve (i.e., $T=A / \lambda$ ). To determine the effect of tortuosity, a low tortuosity arteriole with $T=0.09$ and a high tortuosity arteriole with $T=0.21$ were examined. Each arteriole had the same arc length. The assumption of 2D flow was used for computational efficiency and was expected to give qualitatively similar results and similar relative effects due to RBCs, tortuosity, and MPV compared with three-dimensional (3D) flow. The chosen arteriole diameter, tortuosity indices, and

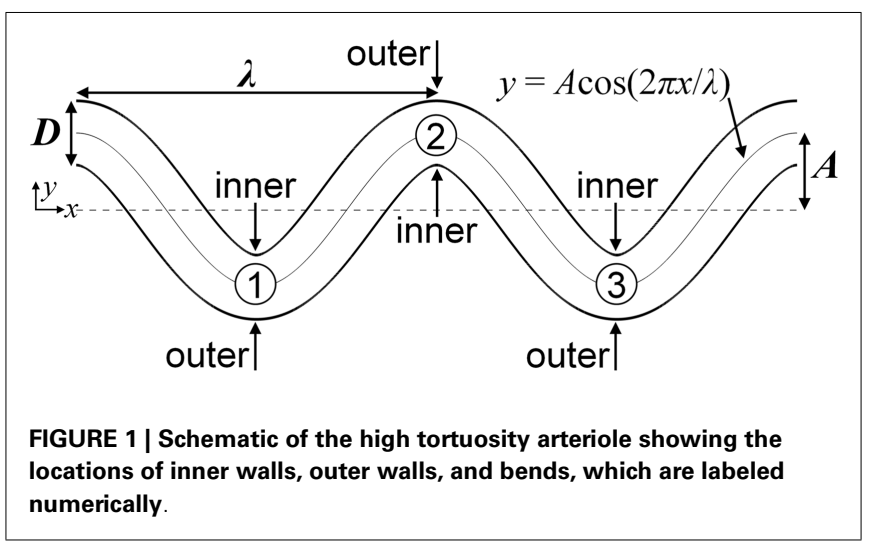

shapes were similar to those observed in tortuous arterioles in humans (e.g., Brown et al., 2002; Sasongko et al., 2012).

Platelets and RBCs were modeled as $3 \mathrm{D}$ spheres for computational efficiency, similar to previous computational studies of thrombus formation that utilized spheres or circles (Mori et al., 2008a; Kamada et al., 2010; Flamm et al., 2011). This approximation was expected to have a small effect on the results and is justified in more detail in the Section "Discussion." In a given simulation, platelets had uniform properties, and RBCs had different uniform properties. To determine the effect of platelet size in pathological states, simulations used three different values of MPV found in humans $(3.8,7.1,15.8 \mathrm{fl})$. The volume of an RBC was set to the physiological value in humans of $94 \mathrm{fl}$. The densities of platelets and RBCs are similar, and so were set to the same cell density $\rho_{\mathrm{c}}=1063 \mathrm{~kg} \mathrm{~m}^{-3}$.

To determine the effects of both platelet distribution and presence of RBCs, three different seeding scenarios were employed to seed cells (i.e., platelets and RBCs) into the arteriole. In all seeding scenarios, cells entered the arteriole at the inlet with an initial velocity equal to the local fluid velocity at the location of the cell centroid, and seeded platelets were initially unactivated. In the whole-lumen-seeded platelets scenario, initial positions of platelet centroids at the inlet followed a pseudorandom probability distribution that excluded the region roughly one platelet radius from the wall such that platelets entered anywhere along the inlet boundary without touching the wall. RBCs were not included in the whole-lumen-seeded platelets scenario. In the near-wall-seeded platelets scenario, initial positions of platelet centroids at the inlet were bounded to regions within $7.5 \mu \mathrm{m}$ of the walls. Centroid positions within these regions followed a pseudorandom probability distribution, without platelets touching the wall at their initial seed locations. This seeding assumed that the platelet distribution at the inlet was that expected in a straight arteriole with whole blood. RBCs, though, were not included in the near-wall-seeded platelets scenario. The RBCs with platelets scenario was the same as the near-wall-seeded platelets scenario, except that RBCs were also seeded into the flow. Positions of RBC centroids were initialized about the center of the arteriole, which spanned the $10-\mu \mathrm{m}$-region in which platelets were not seeded. Centroid positions of RBCs within this region followed a pseudorandom probability distribution. In each seeding scenario after a cell was seeded at the entrance, 
the cell was subjected to no other restrictions on its location or motion.

The number of unactivated platelets that entered the arteriole per unit time (seeding rate) was set such that a physiological timeaveraged platelet count $\left(300,000 \mathrm{~mm}^{-3}\right)$ would be achieved in the absence of platelet adhesion to the walls. Hence, the seeding rate for whole-lumen-seeded platelets was required to be about twice that for near-wall-seeded platelets and for RBCs with platelets. The physiological time-averaged hematocrit was set to $6 \%$. This hematocrit is representative of that in microvessels, which can be much lower than the $\sim 40 \%$ hematocrit of arteries and veins (Keller et al., 1994; Lipowsky, 2005).

Simulations were performed for Newtonian, incompressible, steady, fully developed, 2D flow in the $x-y$ plane. Steady flow was assumed because flow pulsatility is minimal in the microcirculation. Due to low values of hematocrit and volume fraction of platelets, the effects of cells, aggregates, and mural thrombi on the fluid flow were neglected. This assumption is reasonable since the focus of this work was the initial formation of thrombus, rather than later possible occlusion. Centroids of 3D spherical cells remained the $x-y$ plane in the $2 \mathrm{D}$ flow because forces on cells in the $z$-direction were zero, and the only non-zero torques on cells were in the $z$-direction. A Poiseuille velocity profile with typical mean arteriole velocity $U=0.6 \mathrm{~cm} \mathrm{~s}^{-1}$ was imposed on the fluid at the inlet of the arteriole. In a straight arteriole, this velocity profile would yield a wall shear rate $\left|\dot{\gamma}_{0}\right|=1440 \mathrm{~s}^{-1}$, which is in the range of typical values in arterioles (Kroll et al., 1996). Simulations considered blood to be a suspension of RBCs and platelets in plasma. Considered as a single-phase fluid, whole blood is a non-Newtonian fluid with variable apparent viscosity that depends on shear rate, which is mainly due the presence and aggregation of RBCs (Skalak et al., 1981). Rather than treat blood as a continuous fluid, the computational model simulated both plasma and cells, and incorporated a model that accounted for increased drag force on a cell due to increased apparent viscosity from cell crowding in the vicinity of the cell (Di Felice, 1994). Because of these modeling assumptions and the fact that plasma is a Newtonian fluid (Chien et al., 1966), the fluid was chosen to be Newtonian plasma with fluid density $\rho_{\mathrm{f}}=1030 \mathrm{~kg} \mathrm{~m}^{-3}$ and dynamic viscosity $\mu=1.2 \mathrm{cP}$. The parameter values utilized gave a Reynolds number $\left(\operatorname{Re}=\rho_{\mathrm{f}} U D / \mu\right) \sim 0.1$ and particle Stokes numbers $\left(\mathrm{St}=\rho_{\mathrm{c}} d^{2} U / 18 \mu D\right)$ on the order of $10^{-5}$ for platelets and $10^{-4}$ for RBCs, where $d$ is diameter of either a platelet or RBC. Simulation parameters related to physical properties of arterioles, cells, and plasma, as described above, are listed in Table 1.

Measures of thrombosis based on platelet activation, adhesion, and aggregation were determined throughout the simulation time. An aggregate was defined as a group of two or more platelets in contact with each other. A platelet was considered to be part of a mural thrombus if the platelet was in contact with the wall or if it was part of an aggregate that was in contact with the wall. One measure of thrombosis was mural thrombus mass, which was the number of platelets in mural thrombi at a given time multiplied by the mass of a platelet. This measure represented platelets that were in the vessel at the specified time. Another measure was average embolus mass, which was the number of platelets in an aggregate that was not in contact with the wall, averaged over the simulation
Table 1 | Simulation parameters related to physical properties of arterioles, cells, and plasma.

\begin{tabular}{lll}
\hline Parameter & Value & Comments \\
\hline$D$ & $25 \mu \mathrm{m}$ & \\
$T$ (low) & 0.09 & Low tortuosity arteriole, \\
& & $A \approx 16 \mu \mathrm{m}, \lambda \approx 177 \mu \mathrm{m}$ \\
$T$ (high) & 0.21 & High tortuosity arteriole, \\
& & $A \approx 30 \mu \mathrm{m}, \lambda \approx 141 \mu \mathrm{m}$
\end{tabular}

MPV (small platelet) $\quad 3.8 \mathrm{fl} \quad$ Observed in Wiskott-Aldrich syndrome, corresponding to $1.9 \mu \mathrm{m}$ spherical diameter

MPV (normal platelet) $\quad 7.1 \mathrm{fl}$

Physiological value, corresponding to $2.4 \mu \mathrm{m}$ spherical diameter

MPV (large platelet)

$15.8 \mathrm{fl}$

Observed in diabetes, corresponding to $3.1 \mu \mathrm{m}$ spherical diameter

Volume of an RBC

Corresponding to $5.6 \mu \mathrm{m}$ spherical diameter

Platelet count

$300,000 \mathrm{~mm}^{-3} \quad 0.2 \%$ Platelet volume fraction

Platelet seeding rate

$0.67 \mathrm{~ms}^{-1}$

(whole-lumen-seeded)

Platelet seeding rate

(near-wall-seeded)

Hematocrit

$0.34 \mathrm{~ms}^{-1}$

$\rho_{\mathrm{C}}$

$\rho_{f}$

$6 \%$

$1.71 \mathrm{~ms}^{-1} \mathrm{RBC}$ seeding rate

$\mu$

$u$

$\left|\dot{\gamma}_{0}\right|$

$\dot{\gamma}_{\text {crit }}$

$\operatorname{Re}$

St (platelet)

St (RBC)

$1063 \mathrm{~kg} \mathrm{~m}^{-3}$

$1030 \mathrm{~kg} \mathrm{~m}^{-3}$

$1.2 \mathrm{cP}$

$0.6 \mathrm{~cm} \mathrm{~s}^{-1}$

$1440 \mathrm{~s}^{-1}$

$1500 s^{-1}$

0.1

$O\left(10^{-5}\right)$

$-$

time and multiplied by the mass of a platelet. Activation count was defined as the cumulative number of activation events. This measure was a count of the number of platelets that had become activated since the start of the simulation. To compare seeding scenarios that had different platelet seeding rates, activation percent was defined as the percent of seeded platelets that had become activated since the start of the simulation.

Simulations were run to a final time $\left(t_{\mathrm{f}}=1.67 \mathrm{~s}\right)$ at which mural thrombus mass reached a state of statistical equilibrium for cases with the RBCs with platelets scenario. Simulation cases are listed in Table 2. Each simulation was performed as a serial (non-parallel) 
Table 2 | Simulation cases

\begin{tabular}{llll}
\hline Case & Tortuosity & Platelet size & Seeding scenario \\
\hline 1 & Low & Normal & Whole-lumen-seeded platelets \\
2 & Low & Normal & Near-wall-seeded platelets \\
3 & Low & Normal & RBCs with platelets \\
4 & High & Normal & Whole-lumen-seeded platelets \\
5 & High & Normal & Near-wall-seeded platelets \\
6 & High & Normal & RBCs with platelets \\
7 & High & Small & Whole-lumen-seeded platelets \\
8 & High & Small & Near-wall-seeded platelets \\
9 & High & Small & RBCs with platelets \\
10 & High & Large & Whole-lumen-seeded platelets \\
11 & High & Large & Near-wall-seeded platelets \\
12 & High & Large & RBCs with platelets \\
& & &
\end{tabular}

program on a 3.0-GHz node having either four or eight processing cores, with 2 GB of RAM per core. Simulations were run simultaneously on these processing cores. CPU times ranged from 8 to 17 days per simulation.

\section{DISCRETE ELEMENT COMPUTATIONAL MODEL}

The transport, collision, activation, adhesion, and aggregation of hundreds of individual platelets and RBCs were simulated in tortuous arterioles by a mesoscale, discrete element method for blood cells. Details of the computational model have been previously published (Chesnutt and Marshall, 2009a,b; Chesnutt and Han, 2011). A brief description of the model is provided as follows.

The computational model followed the motion of each spherical cell due to interactions with the fluid, vessel walls, and other cells. Activated platelets were subjected to adhesion with the wall and aggregation with each other. RBCs were subjected to aggregation with each other, though shear rates were high enough in this work to suggest disaggregation of RBC aggregates (SchmidSchonbein et al., 1968). In the remainder of this subsection, the term adhesion is used to refer to both adhesion and aggregation.

Cells were modeled as 3D spheres, rather than $2 \mathrm{D}$ circles, so that they possessed mass and could be subjected to the dominant $3 \mathrm{D}$ forces and torques as defined below. Fluid flow was modeled in $2 \mathrm{D}$ to increase computational efficiency. Cell centroids were initialized on the 2D flow $x-y$ plane. As the fluid velocity component in the $z$-direction was zero, and the only non-zero fluid vorticity component was in the $z$-direction, centroids of $3 \mathrm{D}$ cells remained in the $x-y$ plane. Thus, 3D angular and linear momentum equations were reduced to $2 \mathrm{D}$ and were solved for each $3 \mathrm{D}$ cell in $2 \mathrm{D}$ flow, given by

$$
m \frac{d \boldsymbol{v}}{d t}=\boldsymbol{F}_{\mathrm{F}}+\boldsymbol{F}_{\mathrm{A}}, \quad I_{z} \frac{d \Omega_{z}}{d t}=M_{\mathrm{F}, z}+M_{\mathrm{A}, z},
$$

where $m$ is cell mass, $\boldsymbol{v}$ is cell velocity, $I_{z}=(1 / 10) m d^{2}$ is moment of inertia, $\Omega_{z}$ is cell rotation rate, $\boldsymbol{F}_{\mathrm{F}}$ and $M_{\mathrm{F}, z}$ are respectively fluidinduced force and torque on the cell, $\boldsymbol{F}_{\mathrm{A}}$ and $M_{\mathrm{A}, z}$ are respectively force and torque on the cell due to adhesion and collision, and $t$ is time. Fluid-induced force $\boldsymbol{F}_{\mathrm{F}}$ was composed of the dominant fluid-induced forces, which were drag force and added mass force.
Adhesion and collision forces acting on two colliding cells consisted of a force due to elastic deformation of cells $F_{\text {ne }}$, a force due to energy dissipation from collision $F_{\text {nd }}$, and a resistance force $F_{\mathrm{s}}$ due to sliding of one cell over another. Adhesion and collision torques acting on two colliding cells consisted of a torque generated by the sliding resistance force $F_{\mathrm{s}}$ and a torque due to resistance of rolling of one cell over another $M_{\mathrm{r}}$. Adhesion and collision forces and torques were given by

$$
\boldsymbol{F}_{\mathrm{A}}=F_{\mathrm{ne}} \boldsymbol{n}+F_{\mathrm{nd}} \boldsymbol{n}+F_{\mathrm{s}} \boldsymbol{t}_{\mathrm{s}}, \quad M_{\mathrm{A}, z}=\frac{d}{2} F_{\mathrm{s}}\left(\boldsymbol{n} \times \boldsymbol{t}_{\mathrm{s}}\right)+M_{\mathrm{r}}\left(\boldsymbol{t}_{\mathrm{r}} \times \boldsymbol{n}\right),
$$

where $\boldsymbol{n}$ is a unit vector tangent to the line connecting the centroid of the cell to the centroid of the other cell, $\boldsymbol{t}_{\mathrm{s}}$ is a unit vector in the direction of relative motion of the cell surfaces projected onto the plane orthogonal to $\boldsymbol{n}$, and $\boldsymbol{t}_{\mathrm{r}}$ is a unit vector in the direction of rolling velocity of the cell. Forces (drag force, added mass force, $\left.F_{\text {nd }}, F_{\mathrm{s}}\right)$ and torques $\left(M_{\mathrm{F}, z}, M_{\mathrm{r}}\right)$ were determined using equations given in the literature. Elastic deformation force $F_{\text {ne }}$ was determined, as described below, based on the theory of Johnson et al. (1971).

Upon collision, cells were assumed to retain their shapes except within a flattened circular region of contact, in accord with the model of cell-cell contact of Bell et al. (1984). Receptor-ligand bonds that formed within the contact region were modeled as springs following (Bell et al., 1984). Under our model assumptions, receptor-ligand binding took a form that was mathematically analogous to van der Waals adhesion with a time-dependent adhesive surface energy density $\alpha(t)$ given by

$$
\alpha(t)=-\frac{\sigma}{2}\left(x_{\mathrm{b}}-x_{\mathrm{e}}\right)^{2} \int_{0}^{1} N_{\mathrm{b}}\left(t-t_{0}(s)\right) s d s,
$$

where $\sigma$ is spring constant, $x_{\mathrm{b}}$ is length of the bond, $x_{\mathrm{e}}$ is equilibrium length of the bond, $N_{\mathrm{b}}$ is number density of bonds, and $s$ is radial position with the contact region divided by the radius of the contact region. The variation of $N_{\mathrm{b}}$ with time was given by a kinetics equation of Bell (1978) that accounted for forward and reverse reactions of bond formation given by

$$
\frac{d N_{\mathrm{b}}}{d t}=k_{\mathrm{f}}\left(N_{\mathrm{L} 0}-N_{\mathrm{b}}\right)\left(N_{\mathrm{R} 0}-N_{\mathrm{b}}\right)-k_{\mathrm{r}} N_{\mathrm{b}}
$$

where $k_{\mathrm{f}}$ and $k_{\mathrm{r}}$ are respectively forward and reverse reaction rate coefficients, and $N_{\mathrm{L} 0}$ and $N_{\mathrm{R} 0}$ are respectively initial ligand and receptor densities. The reaction rate coefficients were given by Dembo et al. (1988)

$$
\begin{aligned}
& k_{\mathrm{f}}=k_{\mathrm{f} 0} \exp \left[-\frac{\sigma_{\mathrm{ts}}\left(x_{\mathrm{b}}-x_{\mathrm{e}}\right)^{2}}{2 k \bar{T}}\right], \\
& k_{\mathrm{r}}=k_{\mathrm{r} 0} \exp \left[\frac{\left(\sigma-\sigma_{\mathrm{ts}}\right)\left(x_{\mathrm{b}}-x_{\mathrm{e}}\right)^{2}}{2 k \bar{T}}\right],
\end{aligned}
$$

where $k_{\mathrm{f} 0}$ and $k_{\mathrm{r} 0}$ are respectively initial forward and reverse equilibrium reaction rates, $\sigma_{\mathrm{ts}}$ is transition state spring constant, $k$ is the Boltzmann constant, and $\bar{T}$ is absolute temperature. Number 
density of bonds in Eq. 4 was obtained using the solution given by Chesnutt and Marshall (2009a). For simulations in the current work, the time scale for bond formation was much less than the time scale for elastic response of two colliding cells. Thus, number density of bonds was approximated by its equilibrium value $N_{\mathrm{b}}(\infty)$, such that adhesive surface energy density simplified to a constant value, given by

$$
\alpha(t)=-\frac{\sigma}{4}\left(x_{\mathrm{b}}-x_{\mathrm{e}}\right)^{2} N_{\mathrm{b}}(\infty),
$$

where calculations yielded $\alpha(t)=-4 \times 10^{-1} \mathrm{dyn} \mathrm{cm}^{-1}$ for platelets and $-1 \times 10^{-4} \mathrm{dyn}^{-1}$ for RBCs.

Because of the analogy with van der Waals adhesion, we used the theory of Johnson et al. (1971) for adhesive elastic particles to approximate elastic deformation forces acting between two colliding cells, given by

$$
\begin{aligned}
\frac{F_{\mathrm{ne}}}{F_{\mathrm{c}}} & =4\left(\frac{a}{a_{0}}\right)^{3}-4\left(\frac{a}{a_{0}}\right)^{3 / 2}, \\
\frac{\delta_{\mathrm{n}}}{\delta_{\mathrm{c}}} & =6^{1 / 3}\left[2\left(\frac{a}{a_{0}}\right)^{2}-\frac{4}{3}\left(\frac{a}{a_{0}}\right)^{1 / 2}\right],
\end{aligned}
$$

where $a(t)$ is contact region radius, and $\delta_{\mathrm{n}}$ is normal overlap distance of cell surfaces. Critical force $F_{\mathrm{c}}$ and critical overlap $\delta_{\mathrm{c}}$ can be written in terms of contact region radius at equilibrium $a_{0}$ as

$$
F_{\mathrm{c}}=3 \pi \alpha R, \quad \delta_{\mathrm{c}}=\frac{a_{0}^{2}}{2\left(6^{1 / 3}\right) R}, \quad a_{0}=\left(\frac{9 \pi \alpha R^{2}}{E}\right)^{1 / 3}
$$

where $R$ and $E$ are respectively effective radius and effective elastic modulus of the two cells, with adhesive surface energy density $\alpha$ given by Eq. 6 .

Mechanisms of platelet aggregation and adhesion differ depending on shear rate (Jackson et al., 2009). As maximum shear rates in the current work ranged from about $1800-3100 \mathrm{~s}^{-1}$, platelets were assumed to adhere to von Willebrand factor immobilized on the subendothelium through glycoproteins GPIb or GPIIb-IIIa, and to aggregate with each other through bridging of GPIb or GPIIb-IIIa by plasma von Willebrand factor (Ikeda et al., 1991; Kroll et al., 1996; Konstantopoulos et al., 1997; Shankaran et al., 2003). At low shear rates, RBCs aggregate with each other, while at high shear rates these aggregates break up. Though different theories of the mechanisms of RBC aggregation exist (e.g., Chien and Jan, 1973; Neu and Meiselman, 2002), models of receptor-ligand binding of RBCs used in computational studies have produced aggregation behavior similar to experimental observations (Bagchi et al., 2005; Chesnutt and Marshall, 2009a, 2010). Hence, in the current work, RBC aggregation was assumed to occur through receptor-ligand binding.

Typical ranges of values obtained from the literature for parameters in Eqs 4 and 5, which vary widely for different cell adhesion problems, are given in Table 3, along with values used in simulations. Few values of parameters in Table 3 are known specifically for platelet adhesion or aggregation. We selected the density of platelet receptors and other parameters to achieve an effective
Table 3 | Values of parameters related to receptor-ligand binding used in simulations and ranges of experimental values.

\begin{tabular}{llll}
\hline Parameter (units) & $\begin{array}{l}\text { Value used in } \\
\text { simulations for } \\
\text { platelets }\end{array}$ & $\begin{array}{l}\text { Value used in } \\
\text { simulations } \\
\text { for RBCs }\end{array}$ & $\begin{array}{l}\text { Range of } \\
\text { experimental } \\
\text { values }\end{array}$ \\
\hline$k_{\mathrm{fO}}\left(\mathrm{cm}^{2} \mathrm{~s}^{-1}\right)$ & $10^{-7}$ & $10^{-7}$ & $10^{-12}$ to $10^{-7 \mathrm{a}}$ \\
$k_{\mathrm{r} 0}\left(\mathrm{~s}^{-1}\right)$ & $10^{-5}$ & $10^{-5}$ & $10^{-5}$ to $10^{\mathrm{b}}$ \\
$N_{\mathrm{LO}}, N_{\mathrm{RO}}\left(\mathrm{cm}^{-2}\right)$ & $1.115 \times 10^{12}$ & $4.798 \times 10^{9}$ & $10^{9}$ to $10^{12 \mathrm{c}}$ \\
$x_{\mathrm{e}}(\mathrm{nm})$ & 20 & 20 & $5-50^{\mathrm{d}}$ \\
$x_{\mathrm{b}}(\mathrm{nm})$ & 29 & 29 & 0 to length at \\
& & & which bond breaks \\
$\sigma(\mathrm{dyn} \mathrm{cm})$ & 1.98 & 2.2 & $0.01-10^{\mathrm{e}}$ \\
$\sigma_{\mathrm{ts}}\left(\mathrm{d} y \mathrm{nn} \mathrm{cm} \mathrm{cm}^{-1}\right)$ & -5 & -5 & -5 to $5^{\dagger}$ \\
$\bar{T}(\mathrm{~K})$ & 310 & 310 & - \\
\hline
\end{tabular}

a From Hammer and Apte (1992).

${ }^{b}$ From Bell (1978), Ward and Hammer (1993).

${ }^{c}$ From Dembo etal. (1988), Doggett etal. (2002), Reininger et al. (2006), Michelson (2007), Fogelson and Guy (2008).

${ }^{d}$ From Bell et al. (1984), Evans and Leung (1984), Dembo et al. (1988).

eFrom Dembo et al. (1988), Chtcheglova et al. (2004).

${ }^{f}$ From Dembo et al. (1988).

spring constant for adhesion and aggregation of a platelet that was of the same order of magnitude as that determined in a previous study. Specifically, the effective spring constant for a normal sized platelet at the equilibrium contact region radius, calculated as $\sigma \pi a_{0}^{2} N_{\mathrm{b}}(\infty)$, was $34 \mathrm{Nm}^{-1}$. This value compared well with the platelet effective spring constant $50 \mathrm{Nm}^{-1}$, which was determined by Filipovic et al. (2008b) from computational simulations by comparison with experiments. Parameters for RBCs were chosen to obtain the constant adhesive surface energy density $\alpha=-1 \times 10^{-4} \mathrm{dyn}_{\mathrm{cm}}^{-1}$, which previously showed good agreement with in vitro aggregation experiments (Chesnutt and Marshall, 2009a).

\section{SHEAR-INDUCED PLATELET ACTIVATION MODEL}

We used our model of shear-induced platelet activation as in Chesnutt and Han (2011), which was shown in simulations to initiate thrombosis at inner walls in agreement with previous in vivo experiments. This activation model ensured the assumption that a platelet cannot become activated by a physiological shear rate or shear stress, irrespective of the amount of time a platelet is subjected to physiological shear stress. As well, this activation model clearly indicated the point at which an individual platelet became activated. These two attributes of our activation model are not necessarily apparent in other existing models that also account for exposure time to shear stress.

Our model assumed that a platelet became activated if it experienced a shear rate above a critical shear rate $\left(\dot{\gamma}_{\text {crit }}\right)$ defined as $\dot{\gamma}_{\text {crit }}=f\left|\dot{\gamma}_{0}\right|$, where $f$ is a scaling factor greater than unity. We assumed a critical shear stress of $\tau=60 \mathrm{dyn}^{-2}$ to determine critical shear rate. With approximate apparent viscosity of whole blood $\mu_{\mathrm{b}}=4 \mathrm{cP}$, the critical shear stress corresponded to a critical shear rate $\dot{\gamma}_{c r i t}=\tau / \mu_{\mathrm{b}}=1500 \mathrm{~s}^{-1}$. Hence, the scaling factor was chosen to be $f=1.042$. The critical shear stress was chosen based 
on in vitro experiments with whole blood and platelet rich plasma, which showed that shear-induced platelet activation and aggregation occurred at shear stresses that ranged from 15 to $30 \mathrm{dyn} \mathrm{cm}^{-2}$ (Chow et al., 1992; Konstantopoulos et al., 1995, 1997; Shankaran et al., 2003). Although some of these experiments also showed large increases in platelet activation and aggregation as shear stresses surpassed 75-140 dyn $\mathrm{cm}^{-2}$, our critical shear stress was chosen to illustrate trends in the early stage of thrombus formation with respect to changes in shear stress due to tortuosity.

To account for the presence of chemical agonists released by activated platelets, the model assumed a platelet became activated if it contacted another activated platelet, similar to the model of Kamada et al. (2010). In our simulations, only activated platelets were subjected to adhesion and aggregation, and activation was considered irreversible.

\section{RESULTS}

\section{EFFECTS OF RBCs AND TORTUOSITY WITH PLATELETS OF NORMAL SIZE}

In both arterioles, maximum shear rates exceeded the wall shear rate that would occur in a straight arteriole $\left(\left|\dot{\gamma}_{0}\right|=1440 \mathrm{~s}^{-1}\right)$ and the critical shear rate for activation $\left(\left|\dot{\gamma}_{\text {crit }}\right|=1500 \mathrm{~s}^{-1}\right)$. Maximum shear rates were 1764 and $3099 \mathrm{~s}^{-1}$ in the low and high tortuosity arterioles, respectively. The highest shear rates occurred at inner walls in both arterioles (Figure 2), and fluid velocities in these regions were higher for the high tortuosity arteriole (not shown).

Cases with platelets of normal size for the two tortuosity indices and three seeding scenarios (Cases 1-6) are presented in this subsection. In each case, platelets first became activated at locations where shear rates were greater than the critical shear rate (critical shear regions) of the first and second bends. The first few mural thrombi formed on the inner walls of these two bends in some cases (Cases 2, 4, 6), and in other cases formed downstream of these two bends before reaching the third bend (Cases 1, 3, 5). After the onset of a thrombus at inner walls of the first or second bend, the thrombus first grew along the wall in the downstream direction and then later in the upstream direction. Throughout the simulations in each case, mural thrombi continued to form along the walls at other locations, as shown at the final time in Figure 3. For cases with RBCs, almost immediately after the first few mural thrombi formed, RBC collisions with the thrombi caused thrombi to roll along the wall. Throughout the simulations with RBCs, $\mathrm{RBC}$ collisions caused thrombi to continually roll along the wall and exit, generally in a single layer of platelets that could contain 10 or more platelets. For cases without RBCs, thrombi did not roll along the walls, except for one activated platelet and one three-platelet mural thrombus in Case 5.

For each case, emboli continually detached as mural thrombi grew into the flow (away from the wall), with some emboli reattaching on walls downstream. A single layer of platelets remained attached to the wall just after an embolus detached from a mural thrombus. For cases without RBCs, emboli consisted of an average of 8.4-8.9 platelets, with a maximum of 49 platelets. However, with RBCs, emboli were smaller and consisted of an average of 2.6 platelets, with a maximum of 6 platelets.

Activation count initially became larger for cases with RBCs compared with cases with only near-wall-seeded platelets, for a given tortuosity index (Figures 4A,B). This promotion of platelet activation due to RBCs lasted for a longer time in the high
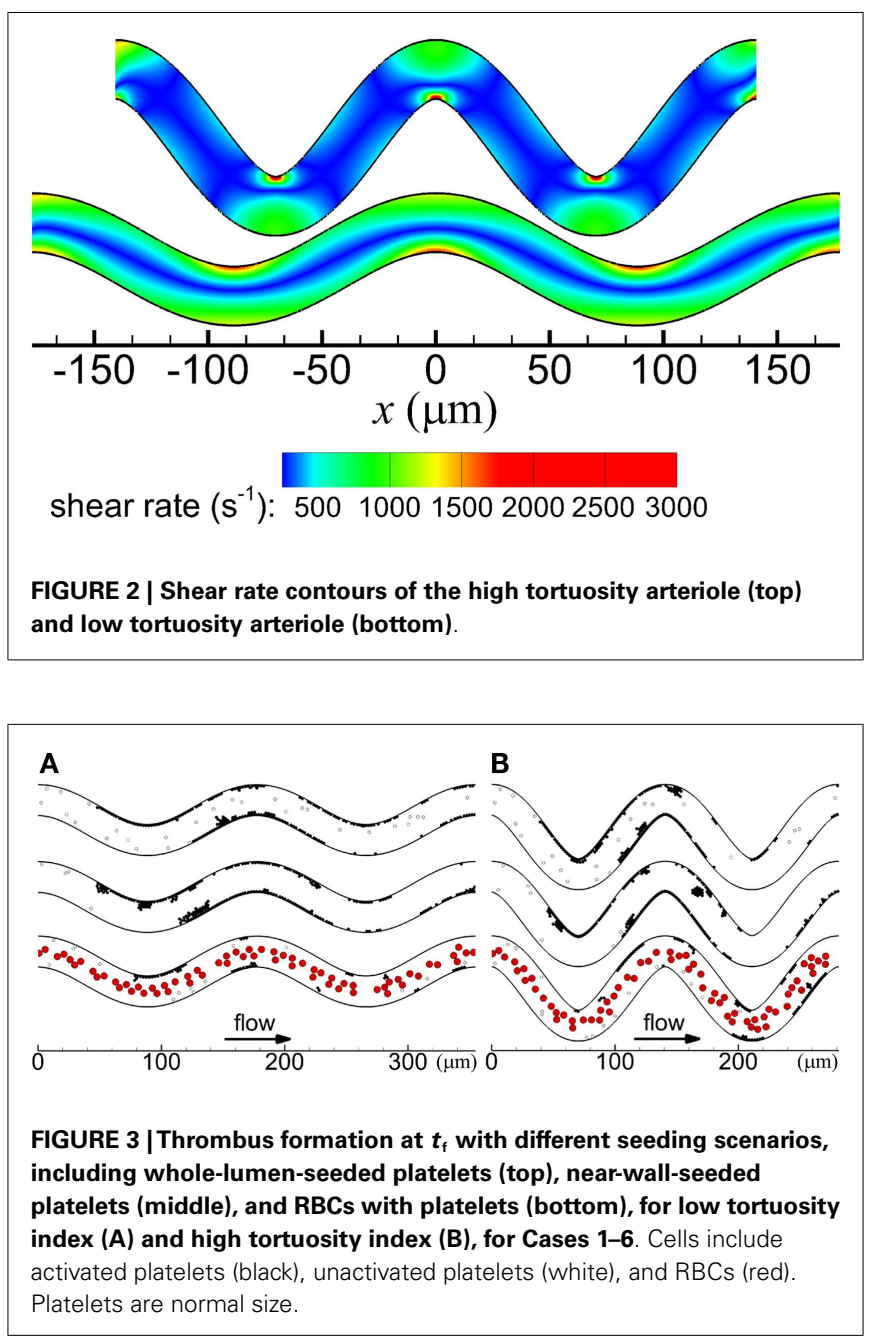

tortuosity arteriole $(\sim 0.6 \mathrm{~s})$ than in the low tortuosity arteriole $(\sim 0.2 \mathrm{~s})$. However, after this short time, activation count became smaller with RBCs compared to near-wall-seeded platelets without RBCs. Due to the higher seeding rate with whole-lumenseeded platelets, activation count for this seeding scenario became larger than the near-wall-seeded platelets scenario and the RBCs with platelets scenario for both tortuous arterioles, as shown in Figure 4C for the high tortuosity arteriole. The low tortuosity arteriole showed similar results to those in Figure 4C, except that activation counts at the final time were slightly smaller at 623,517 , and 462 , with whole-lumen-seeded platelets, near-wallseeded platelets, and RBCs with platelets, respectively. These same values for the high tortuosity arteriole were 654, 532, and 490 . However, activation percent was lowest at most times with wholelumen-seeded platelets (Figure 5), due to the seeding of some platelets near the center of the arteriole.

For a given tortuosity index at any given time after $0.23 \mathrm{~s}$ at most, mural thrombus mass was smallest with RBCs compared to cases without RBCs, and about the same compared between cases without RBCs (Figure 6). Toward the end of the simulations, mural thrombus mass was generally larger for the low (versus high) tortuosity arteriole in the presence of RBCs, but generally smaller for the low tortuosity arteriole in the absence of RBCs. 

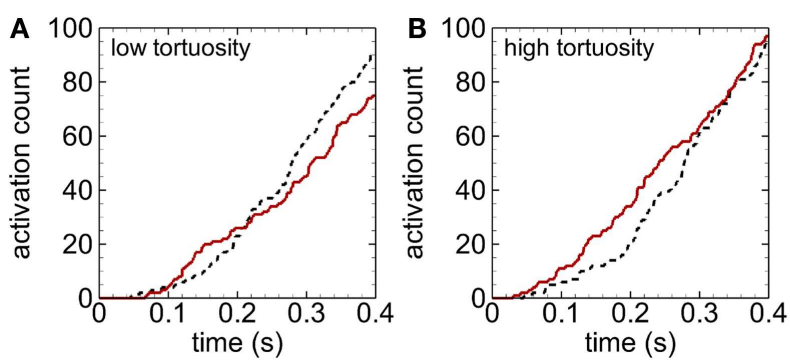

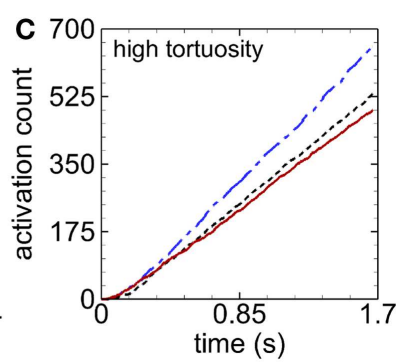

FIGURE 4 | Time variation of activation count during an early stage, for the low tortuosity (A) and high tortuosity (B) arterioles; and during the entire simulation time for the high tortuosity arteriole (C), for Cases 2-6.
Seeding scenarios include whole-lumen-seeded platelets (dashed-dotted line), near-wall-seeded platelets (dashed line), and RBCs with platelets (solid line). Platelets are normal size.
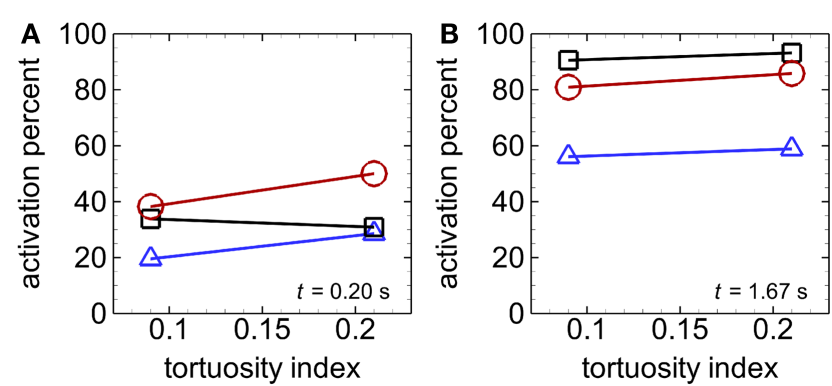

FIGURE 5 | Activation percent versus tortuosity index, with whole-lumen-seeded platelets (triangles), near-wall-seeded platelets (squares), and RBCs with platelets (circles), at an early time $\boldsymbol{t}=0.2 \mathrm{~s}$ (A) and the final time $\boldsymbol{t}_{\mathrm{f}}=\mathbf{1 . 6 7} \mathrm{s}(\mathrm{B})$, for Cases 1-6. Platelets are normal size.
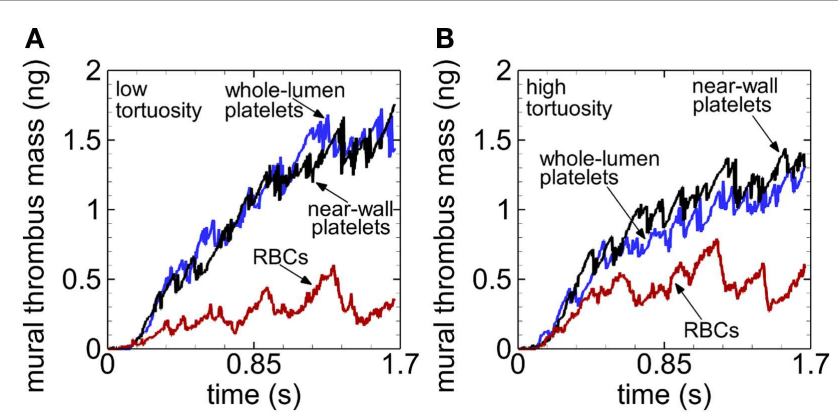

FIGURE 6 | Time variation of mural thrombus mass, with whole-lumen-seeded platelets (blue line), near-wall-seeded platelets (black line), and RBCs with platelets (red line), for the low tortuosity arteriole (A) and high tortuosity arteriole (B), for Cases 1-6. Platelets are normal size.

\section{EFFECTS OF RBCs AT DIFFERENT PLATELET SIZES}

Cases with platelets of different sizes (MPVs) for the three seeding scenarios in the high tortuosity arteriole (Cases 4-12) are presented in this subsection. In these cases, the first few mural thrombi formed either at the inner walls of the first and second bends or on walls downstream of bends after platelet activation at the first and second bends. The location of formation of the first few mural thrombi did not have a clear relationship with

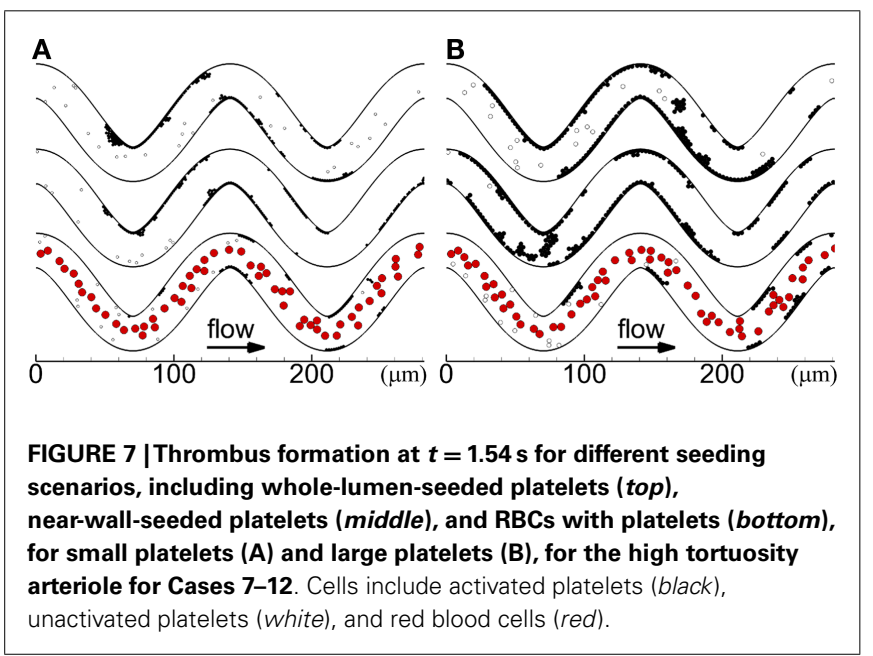

MPV or seeding scenario. Similar to the cases of normal MPV, RBC collisions with abnormal MPV caused mural thrombi to roll along walls and exit the arteriole. Additionally, without RBCs, most cases with abnormal MPV exhibited a small amount of rolling of thrombi, with the large MPV case exhibiting some rolling of large groups of platelets, though less than cases with RBCs. Locations of cells at the end of the simulations are shown for abnormal MPV in Figure 7.

Emboli continually detached from mural thrombi for each case, with some emboli reattaching on walls downstream. Emboli in the absence of RBCs consisted of an average of 6.6-9.5 platelets (maximum of 37 platelets), and in the presence of RBCs an average of 2.3-2.8 platelets (maximum of 7 platelets) (Figure 8A). For a given seeding scenario, although the average number of platelets per embolus decreased with increasing MPV, average embolus mass increased with increasing MPV (Figure 8).

With near-wall-seeded platelets of large MPV, mural thrombi at the first bend eventually grew upstream along the wall and reached the inlet boundary at $t=1.54 \mathrm{~s}$. In this case, locations of new nearwall-seeded platelets could not be seeded into the flow, and so simulations were analyzed at the time when thrombi first blocked the inlet near the wall, at $t=1.54 \mathrm{~s}$, instead of the final simulation time $t=1.67 \mathrm{~s}$. In future studies, the computational model can be modified to seed near-wall-seeded platelets toward the center of 
the arteriole in the event that locations near the walls are occupied by thrombi. Nevertheless, at time $t=1.54 \mathrm{~s}$, mural thrombus mass had reached a state of statistical equilibrium for cases with the RBCs with platelets scenario so that results were expected to be valid. In cases without RBCs, mural thrombi at inner walls of the first and second bends grew in both the upstream and downstream directions. With RBCs, the small MPV case showed slight upstream growth of mural thrombi at the first and second bends before thrombi began to roll along the wall, while the large MPV case showed no upstream growth before thrombi began to roll along the wall.

Unlike the case with normal MPV near the beginning of the simulation, activation count with abnormal MPV was not clearly larger with RBCs compared to only near-wall-seeded platelets. As simulations progressed, activation count was largest for the case with whole-lumen-seeded platelets, followed by the case with near-wall-seeded platelets, and smallest for the case with RBCs, for a given MPV (e.g., Figure 9A). Activation percent at the end of the simulation was smallest for the case with whole-lumenseeded platelets due to possible seeding in the center of the arteriole (Figure 9B). At the final time, activation count (and activation percent) increased as MPV increased for a given seeding scenario.
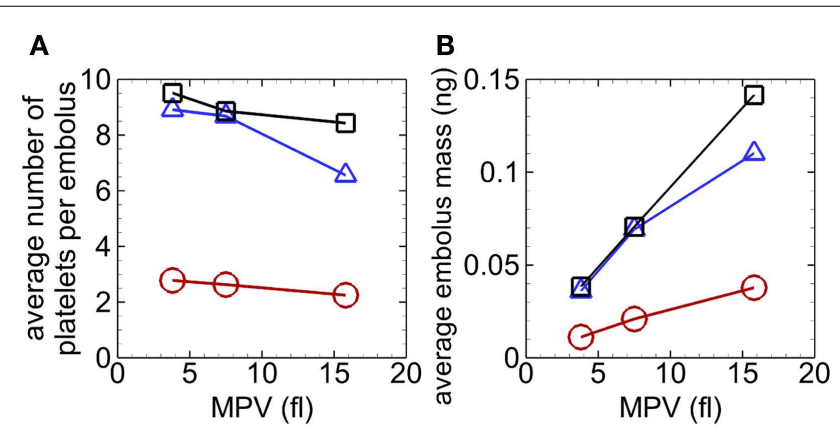

FIGURE 8 | Average number of platelets per embolus versus MPV (A) and average embolus mass versus MPV (B), with whole-lumenseeded platelets (triangles), near-wall-seeded platelets (squares), and RBCs with platelets (circles), for the high tortuosity arteriole for Cases 4-12. Values were averaged over the entire simulation time.
For a given MPV, mural thrombus mass increased with time with similar values for the two cases without RBCs and was smallest with RBCs. The trends with abnormal MPV (not shown) were similar to those with normal MPV (Figure 6B), except that values with small MPV were generally smaller and values with large MPV were generally larger, at a given time for a given seeding scenario. For example, values at the final time were at most 53\% lower with small MPV (0.3-0.8 ng) and at most 176\% higher with large MPV $(1.0-3.6 \mathrm{ng})$. Though at the final time for a given seeding scenario, for cases with abnormal MPV compared with normal MPV, the number of platelets in mural thrombi did not vary as much as mural thrombus mass nor it have as clear a relationship with MPV (Figure 9C). Final numbers of platelets in mural thrombi with abnormal platelets varied from $23 \%$ lower to $25 \%$ higher than with normal platelets, depending on MPV and seeding scenario.

\section{DISCUSSION}

\section{RBCs, MPV, AND TORTUOSITY AFFECT THROMBUS FORMATION}

Current results suggested that for normal sized platelets at the onset of thrombosis, RBCs collided with unactivated platelets to push them closer to walls where critical shear regions or mural thrombi were located. After a very short time, this effect was likely mitigated by formation of large enough mural thrombi that unactivated platelets could easily contact. Later, RBC collisions caused mural thrombi to roll out of the arteriole for all platelet sizes, which reduced platelet activation events, mural thrombus mass, and embolus sizes. Collisions of RBCs with mural thrombi caused emboli to detach before mural thrombi could grow as large as in the case without RBCs. However, without RBCs, mural thrombi were able to grow larger toward the vessel center where larger emboli were detached from mural thrombi by larger fluid forces, in combination with collisions with other platelets. The overall effect of $\mathrm{RBC}$ on thrombus formation in tortuous arterioles may be to reduce the occurrence of thrombotic occlusion of the arteriole or of vessels downstream.

Results of the two seeding scenarios without RBCs indicated that thrombosis (and hemostasis in the case of injury) would proceed in a similar manner, but would be more efficient with near-wall-seeded platelets in a tortuous arteriole, as also expected in a straight vessel. Because the near-wall-seeded platelets scenario virtually excluded collisions of RBCs within the arteriole

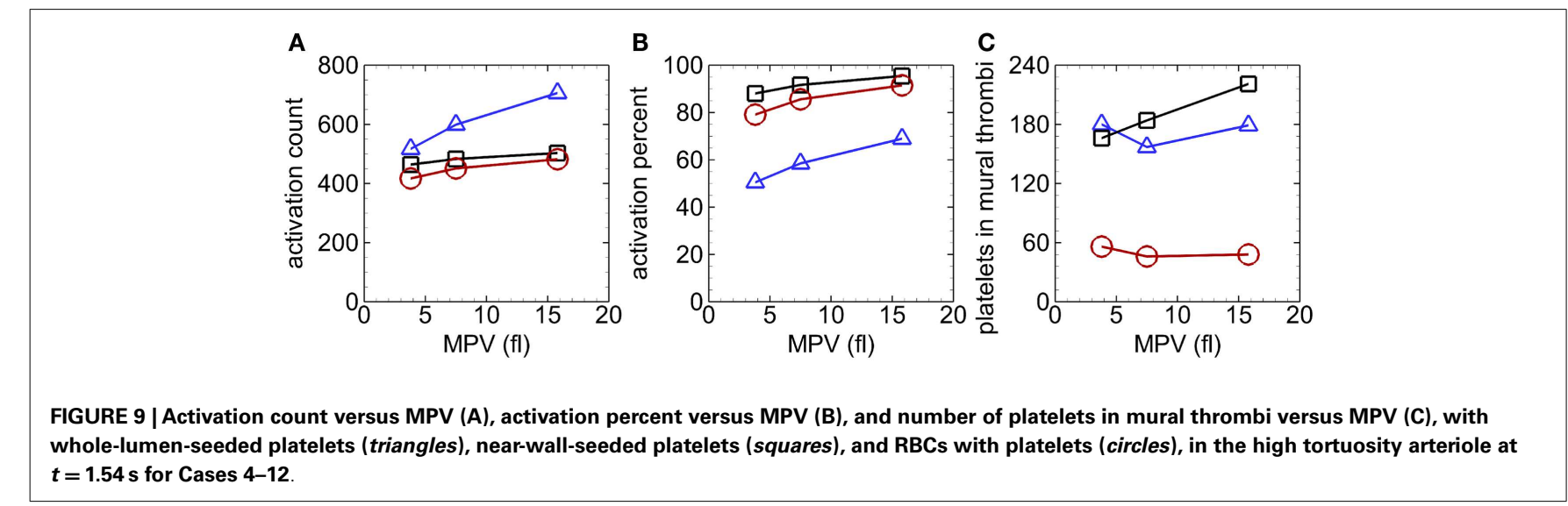


while it mimicked the effect of RBCs on the concentration profile of platelets at the inlet, a partial effect of RBCs would be to increase the efficiency of thrombosis and hemostasis in tortuous arterioles. However, because the RBCs with platelets scenario (i.e., near-wall-seeded platelets scenario with the effect of RBC collisions) caused less mural thrombus mass and smaller emboli sizes, our results suggested that RBCs would increase the efficiency of thrombus initiation, but reduce the risk of thrombotic occlusion due to arteriole tortuosity.

Our work suggested that, due to their small diameter, platelets of small MPV initially had a lower probability of collision with RBCs that could push them toward walls, and so RBCs did not increase activation count initially, as in the case of normal MPV. Moreover, RBCs did not increase activation count initially in the case of large MPV, which was possibly due to platelets of large mass undergoing smaller rebound velocities after collisions with RBCs that could push them toward walls. Our study showed that increased MPV yielded increased activation count at the final time for a given seeding scenario, including the two scenarios without RBCs and the scenario with RBCs. This result agreed with our previous study with whole-lumen-seeded platelets without RBCs (Chesnutt and Han, 2013), which was demonstrated at the tortuosity indices used in the current study, in addition to two intermediate tortuosity indices. Larger MPV may promote thrombosis through larger mural thrombus mass and larger emboli that would be more likely to occlude the arteriole or vessels downstream.

Our results revealed that tortuosity could generate high enough shear rates to induce platelet activation. Our results also suggested that, in the absence of RBCs, mural thrombi remained more intact in the arteriole of low tortuosity than in the arteriole of high tortuosity, likely due to forces at inner walls of bends (fluid and collision forces) that were lower in the arteriole of low tortuosity. Therefore, the fact that mural thrombus mass was smaller in the presence of RBCs in the arteriole of low tortuosity must be attributed to one or more of the following presumed events: more RBC collisions with mural thrombi, higher RBC collision forces with mural thrombi, or more rolling of mural thrombi out of the vessel at low tortuosity. Additional simulations to specifically measure individual collision events and collision forces or to virtually exclude specific events, such as thrombus rolling, may elucidate reasons for these results in future studies.

Our simulation of the process of thrombus formation yielded results that were similar to previous in vivo, in vitro, and computational studies. For example, thrombus formation was initiated at inner walls in some cases. This result agreed with a previous in vivo study in rats, which showed thrombosis was initiated at inner walls of curved venules due to high shear stress and high shear stress gradient in those regions (Liu et al., 2008). Another study with porcine whole blood perfused through glass stenoses that were coated with collagen showed that platelets initially adhered at the downstream end of the stenosis with some adhering upstream of the apex of the stenosis (Para et al., 2011). Later, thrombus formed both upstream and downstream of the stenosis apex. Although the very high shear rates in this previous study $\left(\sim 100,000 \mathrm{~s}^{-1}\right)$ indicated that platelet adhesion and aggregation occurred through different mechanisms than in our study (see Materials and Methods), the process of initial thrombus formation was similar to our study, in that thrombi that formed at the region of highest shear grew downstream first and then upstream later.

In a previous $2 \mathrm{D}$ computational study in simple shear flow with shear rate of $1000 \mathrm{~s}^{-1}$, platelets became activated by an injured wall segment to form a thrombus (Mori et al., 2008a). RBCs caused the thrombus to grow more along the wall than into the flow, and caused larger a thrombus mass. Our simulations agreed with the former result but not the latter. The discrepancy may be partially due to differences in the width of the region in which platelets were seeded, which was larger in the previous study $(17 \mu \mathrm{m})$ than in our study $(7.5 \mu \mathrm{m})$. Our smaller seeding width provided less space for thrombus to grow away from the wall and more opportunities for RBCs to collide with the layer of platelets adhered to the wall, causing thrombus to roll and exit the vessel. However, in the previous study of Mori et al. (2008a), RBCs were farther away from the thrombus base such that the thrombus was several platelet layers deep, and RBCs collided with platelets to push them toward the thrombus, rather than detach them from the wall.

A recent 3D computational study simulated individual platelets and deformable RBCs in straight cylindrical tubes of diameters between about 12 and $15 \mu \mathrm{m}$, with length $40 \mu \mathrm{m}$ and Re about 0.01-0.02 (Kamada et al., 2012), which were smaller vessels and flow rates than in our study. In these previous simulations with and without RBCs, platelets became activated due to an injured wall segment, adhered to the injured wall, and initiated thrombus formation. The mural thrombus then grew downstream, with some platelets detaching from the thrombus due to collisions with $\mathrm{RBC}$ or due to fluid forces. These initial processes of thrombus formation and effects of RBC collisions and fluid forces were in agreement with our results, though thrombosis was initiated by high shear stress in our study, rather than injury. However, the presence or absence of RBCs did not change thrombus height, area of injured wall covered by the thrombus, or number of platelets in the thrombus in this previous study. As noted by the authors, this result was due to the deformation of RBCs during collision with the thrombus. In contrast, our study assumed rigid RBCs and found smaller mural thrombus mass in the presence of RBCs. However, our Reynolds number $(\operatorname{Re}=0.1)$ was 10 times higher than in the previous study, such that if deformable RBCs were used in our study, we would have observed larger RBC velocities that might produce large enough RBC collision forces to detach platelets from mural thrombi. Differences in results between the presence and absence of RBCs would be less pronounced with deformable RBCs than with rigid RBCs, but we would expect an effect of RBCs.

\section{MODEL LIMITATIONS}

The model of shear-induced platelet activation did not account for exposure time to shear stress, which has been shown to affect activation in vitro (Hellums, 1994). Our previous work (Chesnutt and Han, 2013) showed that our activation model was either in quantitative or qualitative agreement with a shear-induced platelet activation model that accounted for magnitude of and exposure time to shear stress (Alemu and Bluestein, 2007), depending on the parameters chosen for the model of Alemu and Bluestein.

The model contained various assumptions for computational efficiency. Platelets and RBCs were assumed to be spherical and 
rigid in the absence of collision or adhesion. Although unactivated platelets are discoid, activated platelets are more spheroidal (Michelson, 2007). Hence, the shapes of activated platelets are closer to spheres than disks. As the focus of our work was on thrombus formation by activated platelets, the difference in results due to use of a spherical platelet shape, rather than a spheroidal shape, were expected to be small. RBCs have a resting shape that is a biconcave disk, and can exhibit either a tumbling motion or tanktreading motion in fluid flow depending on shear rate (Bitbol, 1986). As well, RBCs are highly deformable, which is necessary for RBCs to traverse capillaries, which have smaller diameters than RBCs (Skalak et al., 1989). Because our study was simulated in arterioles with diameters larger than capillaries and focused on the effect of the overall presence of RBCs on thrombus formation, the assumption of spherical non-deformable RBCs was expected to have a small effect on the results.

Fluid flow was assumed to be $2 \mathrm{D}$ and unaffected by the presence of cells (one-way coupled). Under these assumptions, our previous work showed that aggregates of cells in $2 \mathrm{D}$ shear flows were quantitatively similar in size and shape to those in 3D flows (Chesnutt and Marshall, 2010), and that thrombosis was initiated at the same locations in 2D channel flows as in vivo (Chesnutt and Han, 2011). Also, the effects of cells on the fluid were reduced due to the low volume fractions of platelets and RBCs in our simulations. Because this work focused on initial thrombus formation, rather than occlusion, the assumptions of 2D flow and one-way coupling were expected to give qualitatively similar results and similar relative effects due to RBCs, MPV, and tortuosity compared to $3 \mathrm{D}$ flow and two-way coupling.

\section{CLINICAL RELEVANCE}

The physical effects of RBCs, MPV, and tortuosity may compound the chemical factors and other physical factors present in disease states. In the absence of RBCs, our results showed much larger mural thrombus mass and embolus size, which suggested a higher risk of thrombotic complications. This result was in accord with a retrospective study of anemic patients who underwent free flap reconstructive surgery (Hill et al., 2012). Preoperative values of hematocrit and hemoglobin were significantly lower in patients who experienced thrombosis and who experienced flap failure, which was primarily due to thrombosis. The authors proposed that thrombosis could have been partly due to increased turbulence from lower blood viscosity or due to alterations in platelet aggregation and concentrations of coagulation factors. In addition to these factors, our results suggest that fewer RBC collisions due to lower hematocrit might promote thrombosis once thrombi begin to form during free flap procedures in anemic patients.

In cases with RBCs, mural thrombus mass and embolus mass were about double for cases with large platelets compared to cases with normal platelets. Hence, the physical effects of large platelets and microvascular tortuosity in diabetes may be partly responsible for the large incidence of thrombosis, which is also attributed to hypercoagulability in diabetes. Because metformin reduces platelet size in diabetes (Dolasik et al., 2013), this drug may also reduce the risk of thrombosis due to the physical effects of platelet size. This mechanism was speculated in our previous work that neglected
RBCs (Chesnutt and Han, 2013) and is confirmed by the current work that includes RBCs. Mural thrombus mass was about half for cases with small platelets compared to cases with normal platelets, throughout the simulations with RBCs. Assuming a similar effect under conditions of platelet activation due to vessel injury, rather than high shear stress, this physical effect of small platelet size may contribute to the high risk of bleeding observed in WiskottAldrich syndrome, which is also attributed to severely low platelet count and other factors (Ochs et al., 1980).

\section{CONCLUSION AND OUTLOOK}

The computational model simulated individual platelets and RBCs in the microscale processes of shear-mediated thrombus formation in tortuous arterioles. We concluded that RBCs initially promoted platelet activation, but that this effect was quickly reversed as simulations progressed. Collisions of RBCs with mural thrombi yielded reduced mural thrombus mass and embolus mass. As well, a reduction in platelet size resulted in reduced mural thrombus mass and embolus mass, either with or without RBCs. Hence, vessel occlusion may be less likely due to the presence of RBCs and due to smaller platelet size. A smaller mural thrombus mass was observed at the low tortuosity index compared to the high tortuosity index in the presence of RBCs. Our results suggest that the physical mechanisms associated with RBCs, platelet size, and vessel tortuosity play important roles in platelet activation and thrombus formation.

To our knowledge, except for our two previous works (Chesnutt and Han, 2011, 2013), no other computational studies have simulated shear-induced activation of individual platelets in thrombus formation, possibly because previous activation models did not clearly indicate the incident of activation. With the clear occurrence of platelet activation in our computational model, we were able to simulate a large number of platelets in thrombus formation. Our study shed light on mechanical factors involved in tortuosityinduced thrombosis, including the presence of RBCs, platelet size, and microvessel tortuosity. The model serves as a foundation to build upon in future work, for example with improvements such as more physiological shapes of RBCs and platelets and/or 3D fluid flows. Future studies may help determine the risk of thrombotic or bleeding complications due to physical mechanisms associated with RBCs, platelets, and vessel tortuosity, to aid treatment and prevention of these complications in various pathologies.

\section{AUTHOR CONTRIBUTIONS}

Hai-Chao Han and Jennifer K. W. Chesnutt contributed to study conception and design, interpretation of data, and drafting and critical revision of the manuscript. Jennifer K. W. Chesnutt acquired and analyzed data.

\section{ACKNOWLEDGMENTS}

This work was supported by National Institutes of Health National Heart, Lung, and Blood Institute (T32HL007446 to Jennifer K. W. Chesnutt and R01HL095852 to Hai-Chao Han), National Science Foundation (CAREER award \#0644646 to Hai-Chao Han), and Computational Systems Biology Core funded by National Institutes of Health National Institute on Minority Health and Health Disparities (G12MD007591). The content is solely the 
responsibility of the authors and does not necessarily represent the official views of the National Institutes of Health or the National Science Foundation.

\section{REFERENCES}

Aarts, P. A., van den Broek, S. A., Prins, G. W., Kuiken, G. D., Sixma, J. J., and Heethaar, R. M. (1988). Blood platelets are concentrated near the wall and red blood cells, in the center in flowing blood. Arteriosclerosis 8, 819-824. doi:10.1161/01.ATV.8.6.819

Alemu, Y., and Bluestein, D. (2007). Flow-induced platelet activation and damage accumulation in a mechanical heart valve: numerical studies. Artif. Organs 31, 677-688. doi:10.1111/j.1525-1594.2007.00446.x

AlMomani, T., Udaykumar, H. S., Marshall, J. S., and Chandran, K. B. (2008). Microscale dynamic simulation of erythrocyte-platelet interaction in blood flow. Ann. Biomed. Eng. 36, 905-920. doi:10.1007/s10439-008-9478-z

Bagchi, P., Johnson, P. C., and Popel, A. S. (2005). Computational fluid dynamic simulation of aggregation of deformable cells in a shear flow. J. Biomech. Eng. 127, 1070-1080. doi:10.1115/1.2112907

Bell, G. I. (1978). Models for the specific adhesion of cells to cells. Science 200, 618-627. doi:10.1126/science. 347575

Bell, G. I., Dembo, M., and Bongrand, P. (1984). Cell adhesion. Competition between nonspecific repulsion and specific bonding. Biophys. J. 45, 1051-1064. doi:10.1016/S0006-3495(84)84252-6

Bitbol, M. (1986). Red blood cell orientation in orbit C = 0. Biophys. J.49, 1055-1068. doi:10.1016/S0006-3495(86)83734-1

Brown, W. R., Moody, D. M., Challa, V. R., Thore, C. R., and Anstrom, J. A. (2002). Venous collagenosis and arteriolar tortuosity in leukoaraiosis. J. Neurol. Sci. 20, 159-163. doi:10.1016/S0022-510X(02)00283-6

Cambronero, F., Marin, F., Roldan, V., Hernandez-Romero, D., Valdes, M., and Lip, G. Y. (2009). Biomarkers of pathophysiology in hypertrophic cardiomyopathy: implications for clinical management and prognosis. Eur. Heart J. 30, 139-151. doi:10.1093/eurheartj/ehn538

Chesnutt, J. K., and Han, H. C. (2013). Platelet size and density affect shearinduced thrombus formation in tortuous arterioles. Phys. Biol. 10, 056003. doi:10.1088/1478-3975/10/5/056003

Chesnutt, J. K. W., and Han, H. C. (2011). Tortuosity triggers platelet activation and thrombus formation in microvessels. J. Biomech. Eng. 133, 121004. doi: $10.1115 / 1.4005478$

Chesnutt, J. K. W., and Marshall, J. S. (2009a). Blood cell transport and aggregation using discrete ellipsoidal particles. Comput. Fluids 38, 1782-1794. doi:10.1016/j. compfluid.2009.04.002

Chesnutt, J. K. W., and Marshall, J. S. (2009b). Effect of particle collisions and aggregation on red blood cell passage through a bifurcation. Microvasc. Res. 78, 301-313. doi:10.1016/j.mvr.2009.09.003

Chesnutt, J. K. W., and Marshall, J. S. (2010). Structural analysis of red blood cell aggregates under shear flow. Ann. Biomed. Eng. 38, 714-728. doi:10.1007/s10439009-9871-2

Chien, S., and Jan, K. (1973). Ultrastructural basis of the mechanism of rouleaux formation. Microvasc. Res. 5, 155-166. doi:10.1016/0026-2862(73)90068-X

Chien, S., Usami, S., Taylor, H. M., Lundberg, J. L., and Gregerse, M. I. (1966). Effects of hematocrit and plasma proteins on human blood rheology at low shear rates. J. Appl. Physiol. 21, 81-87.

Chow, T. W., Hellums, J. D., Moake, J. L., and Kroll, M. H. (1992). Shear stressinduced von Willebrand factor binding to platelet glycoprotein Ib initiates calcium influx associated with aggregation. Blood 80, 113-120.

Chtcheglova, L. A., Shubeita, G. T., Sekatskii, S. K., and Dietler, G. (2004). Force spectroscopy with a small dithering of AFM tip: a method of direct and continuous measurement of the spring constant of single molecules and molecular complexes. Biophys. J. 86, 1177-1184. doi:10.1016/S0006-3495(04)74192-2

Chu, S. G., Becker, R. C., Berger, P. B., Bhatt, D. L., Eikelboom, J. W., Konkle, B., et al. (2010). Mean platelet volume as a predictor of cardiovascular risk: a systematic review and meta-analysis. J. Thromb. Haemost. 8, 148-156. doi:10.1111/j.1538-7836.2009.03584.x

Dembo, M., Torney, D. C., Saxman, K., and Hammer, D. (1988). The reaction-limited kinetics of membrane-to-surface adhesion and detachment. Proc. R. Soc. Lond. B Biol. Sci. 234, 55-83. doi:10.1098/rspb.1988.0038

Di Felice, R. (1994). The voidage function for fluid-particle interaction systems. Int. J. Multiphas. Flow 20, 153-159. doi:10.1016/0301-9322(94)90011-6
Doggett, T. A., Girdhar, G., Lawshe, A., Schmidtke, D. W., Laurenzi, I. J., Diamond, S. L., et al. (2002). Selectin-like kinetics and biomechanics promote rapid platelet adhesion in flow: the GPIb(alpha)-vWF tether bond. Biophys. J. 83, 194-205. doi:10.1016/S0006-3495(02)75161-8

Dolasik, I., Sener, S. Y., Celebi, K., Aydin, Z. M., Korkmaz, U., and Canturk, Z. (2013). The effect of metformin on mean platelet volume in diabetic patients. Platelets 24, 118-121. doi:10.3109/09537104.2012.674165

Erdem, E., Erdem, D., Dilek, M., Kaya, C., Karatas, A., Kut, E., et al. (2012). Red cell distribution width and mean platelet volume in amyloidosis. Clin. Appl. Thromb. Hemost. doi:10.1177/1076029612462761

Evans, E., and Leung, A. (1984). Adhesivity and rigidity of erythrocyte membrane in relation to wheat germ agglutinin binding. J. Cell Biol. 98, 1201-1208. doi:10.1083/jcb.98.4.1201

Filipovic, N., Kojic, M., and Tsuda, A. (2008a). Modelling thrombosis using dissipative particle dynamics method. Philos. Trans. A Math. Phys. Eng. Sci. 366, 3265-3279. doi:10.1098/rsta.2008.0097

Filipovic, N., Ravnic, D., Kojic, M., Mentzer, S. J., Haber, S., and Tsuda, A. (2008b). Interactions of blood cell constituents: experimental investigation and computational modeling by discrete particle dynamics algorithm. Microvasc. Res. 75, 279-284. doi:10.1016/j.mvr.2007.09.007

Flamm, M. H., Sinno, T., and Diamond, S. L. (2011). Simulation of aggregating particles in complex flows by the lattice kinetic Monte Carlo method. J. Chem. Phys. 134, 034905. doi:10.1063/1.3521395

Fogelson, A. L., and Guy, R. D. (2008). Immersed-boundary-type models of intravascular platelet aggregation. Comput. Methods Appl. Mech. Eng. 197, 2087-2104. doi:10.1016/j.cma.2007.06.030

Gando, S. (2010). Microvascular thrombosis and multiple organ dysfunction syndrome. Crit. Care Med. 38, S35-S42. doi:10.1097/CCM.0b013e3181c9e31d

Guvenc, T. S., Erer, H. B., Ilhan, S., Zeren, G., Ilhan, E., Karakus, G., et al. (2012). Comparison of mean platelet volume values among different causes of pulmonary hypertension. Cardiol. J. 19, 180-187. doi:10.5603/CJ.2012.0031

Hammer, D. A., and Apte, S. M. (1992). Simulation of cell rolling and adhesion on surfaces in shear flow: general results and analysis of selectin-mediated neutrophil adhesion. Biophys. J. 63, 35-57. doi:10.1016/S0006-3495(92)81577-1

Hellums, J. D. (1994). 1993 Whitaker lecture: biorheology in thrombosis research. Ann. Biomed. Eng. 22, 445-455. doi:10.1007/BF02367081

Hill, J. B., Patel, A., Del Corral, G. A., Sexton, K. W., Ehrenfeld, J. M., Guillamondegui, O. D., et al. (2012). Preoperative anemia predicts thrombosis and free flap failure in microvascular reconstruction. Ann. Plast. Surg. 69, 364-367. doi:10.1097/SAP.0b013e31823ed606

Hutchins, G. M., Miner, M. M., and Bulkley, B. H. (1978). Tortuosity as an index of age and diameter increase of coronary collateral vessels in patients after acute myocardial-infarction. Am. J. Cardiol. 41, 210-215. doi:10.1016/0002-9149(78) 90158-3

Ikeda, Y., Handa, M., Kawano, K., Kamata, T., Murata, M., Araki, Y., et al. (1991). The role of von Willebrand factor and fibrinogen in platelet aggregation under varying shear stress. J. Clin. Invest. 87, 1234-1240. doi:10.1172/JCI115124

Jackson, S. P., Nesbitt, W. S., and Westein, E. (2009). Dynamics of platelet thrombus formation. J. Thromb. Haemost. 7, 17-20. doi:10.1111/j.1538-7836. 2009.03401.x

Johnson, K. L., Kendall, K., and Roberts, A. D. (1971). Surface energy and contact of elastic solids. Philos. Trans. R. Soc. Lond. A 324, 301-313. doi:10.1098/rspa.1971. 0141

Kamada, H., Imai, Y., Nakamura, M., Ishikawa, T., and Yamaguchi, T. (2012). Computational analysis on the mechanical interaction between a thrombus and red blood cells: possible causes of membrane damage of red blood cells at microvessels. Med. Eng. Phys. 34, 1411-1420. doi:10.1016/j.medengphy.2012.01.003

Kamada, H., Tsubota, K., Nakamura, M., Wada, S., Ishikawa, T., and Yamaguchi, T. (2010). A three-dimensional particle simulation of the formation and collapse of a primary thrombus. Int. J. Numer. Method Biomed. Eng. 26, 488-500. doi: $10.1002 / \mathrm{cnm} .1367$

Kamada, H., Tsubota, K., Nakamura, M., Wada, S., Ishikawa, T., and Yamaguchi, T. (2011). Computational study on effect of stenosis on primary thrombus formation. Biorheology 48, 99-114. doi:10.3233/BIR-2011-0585

Karino, T., and Goldsmith, H. L. (1984). Role of blood cell-wall interactions in thrombogenesis and atherogenesis - a microrheological study. Biorheology 21, 587-601.

Karpatkin, S. (1978). Heterogeneity of human platelets. VI. Correlation of platelet function with platelet volume. Blood 51, 307-316. 
Keller, M. W., Damon, D. N., and Duling, B. R. (1994). Determination of capillary tube hematocrit during arteriolar microperfusion. Am. J. Physiol. 266, H2229-H2238.

Konstantopoulos, K., Chow, T. W., Turner, N. A., Hellums, J. D., and Moake, J. L. (1997). Shear stress-induced binding of von Willebrand factor to platelets. Biorheology 34, 57-71. doi:10.1016/S0006-355X(97)00004-8

Konstantopoulos, K., Wu, K. K., Udden, M. M., Banez, E. I., Shattil, S. J., and Hellums, J. D. (1995). Flow cytometric studies of platelet responses to shear stress in whole blood. Biorheology 32, 73-93.

Kroll, M. H., Hellums, J. D., McIntire, L. V., Schafer, A. I., and Moake, J. L. (1996). Platelets and shear stress. Blood 88, 1525-1541.

Lipowsky, H. H. (2005). Microvascular rheology and hemodynamics. Microcirculation 12, 5-15. doi:10.1080/10739680590894966

Liu, Q., Mirc, D., and Fu, B. M. (2008). Mechanical mechanisms of thrombosis in intact bent microvessels of rat mesentery. J. Biomech. 41, 2726-2734. doi:10.1016/j.jbiomech.2008.06.013

Mhawech, P., and Saleem, A. (2000). Inherited giant platelet disorders. Classification and literature review. Am. J. Clin. Pathol. 113, 176-190. doi:10.1309/FC4HLM5V-VCW8-DNJU

Michelson, A. D. (2007). Platelets. Boston: Academic Press/Elsevier.

Miyazaki, H., and Yamaguchi, T. (2003). Formation and destruction of primary thrombi under the influence of blood flow and von Willebrand factor analyzed by a discrete element method. Biorheology 40, 265-272.

Mori, D., Yano, K., Tsubota, K., Ishikawa, T., Wada, S., and Yamaguchi, T. (2008a). Computational study on effect of red blood cells on primary thrombus formation. Thromb. Res. 123, 114-121. doi:10.1016/j.thromres.2008.03.006

Mori, D., Yano, K., Tsubota, K., Ishikawa, T., Wada, S., and Yamaguchi, T. (2008b). Simulation of platelet adhesion and aggregation regulated by fibrinogen and von Willebrand factor. Thromb. Haemost. 99, 108-115. doi:10.1160/TH07-08-0490

Neu, B., and Meiselman, H. J. (2002). Depletion-mediated red blood cell aggregation in polymer solutions. Biophys. J. 83, 2482-2490. doi:10.1016/S0006-3495(02) 75259-4

Ochs, H. D., Slichter, S. J., Harker, L. A., Von Behrens, W. E., Clark, R. A., and Wedgwood, R. J. (1980). The Wiskott-Aldrich syndrome: studies of lymphocytes, granulocytes, and platelets. Blood 55, 243-252.

Owen, C. G., Newsom, R. S., Rudnicka, A. R., Barman, S. A., Woodward, E. G., and Ellis, T. J. (2008). Diabetes and the tortuosity of vessels of the bulbar conjunctiva. Ophthalmology 115, e27-e32. doi:10.1016/j.ophtha.2008.02.009

Papanas, N., Symeonidis, G., Maltezos, E., Mavridis, G., Karavageli, E., Vosnakidis, T., et al. (2004). Mean platelet volume in patients with type 2 diabetes mellitus. Platelets 15, 475-478. doi:10.1080/0953710042000267707

Para, A., Bark, D., Lin, A., and Ku, D. (2011). Rapid platelet accumulation leading to thrombotic occlusion. Ann. Biomed. Eng. 39, 1961-1971. doi:10.1007/s10439011-0296-3

Pivkin, I. V., Richardson, P. D., and Karniadakis, G. (2006). Blood flow velocity effects and role of activation delay time on growth and form of platelet thrombi. Proc. Natl. Acad. Sci. U.S.A. 103, 17164-17169. doi:10.1073/pnas.0608546103

Reasor, D. A. Jr., Mehrabadi, M., Ku, D. N., and Aidun, C. K. (2012). Determination of critical parameters in platelet margination. Ann. Biomed. Eng. 41, 238-249. doi:10.1007/s10439-012-0648-7

Reininger, A. J., Heijnen, H. F., Schumann, H., Specht, H. M., Schramm, W., and Ruggeri, Z. M. (2006). Mechanism of platelet adhesion to von Willebrand factor and microparticle formation under high shear stress. Blood 107, 3537-3545. doi:10.1182/blood-2005-02-0618
Sasongko, M. B., Wong, T. Y., Donaghue, K. C., Cheung, N., Jenkins, A. J., Benitez-Aguirre, P., et al. (2012). Retinal arteriolar tortuosity is associated with retinopathy and early kidney dysfunction in type 1 diabetes. Am. J. Ophthalmol. 153, 176-183. doi:10.1016/j.ajo.2011.06.005

Schmid-Schonbein, H., Gaehtgens, P., and Hirsch, H. (1968). On the shear rate dependence of red cell aggregation in vitro. J. Clin. Invest. 47, 1447-1454. doi:10.1172/JCI105836

Shankaran, H., Alexandridis, P., and Neelamegham, S. (2003). Aspects of hydrodynamic shear regulating shear-induced platelet activation and self-association of von Willebrand factor in suspension. Blood 101, 2637-2645. doi:10.1182/blood2002-05- 1550

Skalak, R., Keller, S. R., and Secomb, T. W. (1981). Mechanics of blood flow. J. Biomech. Eng. 103, 102-115. doi:10.1115/1.3138253

Skalak, R., Ozkaya, N., and Skalak, T. C. (1989). Biofluid mechanics. Annu. Rev. Fluid Mech. 21, 167-204. doi:10.1146/annurev.fl.21.010189.001123

Spangler, K. M., Challa, V. R., Moody, D. M., and Bell, M. A. (1994). Arteriolar tortuosity of the white matter in aging and hypertension. A microradiographic study. J. Neuropathol. Exp. Neurol. 53, 22-26. doi:10.1097/00005072-19940100000003

Tokarev, A. A., Butylin, A. A., and Ataullakhanov, F. I. (2011a). Platelet adhesion from shear blood flow is controlled by near-wall rebounding collisions with erythrocytes. Biophys. J. 100, 799-808. doi:10.1016/j.bpj.2010.12.3740

Tokarev, A. A., Butylin, A. A., Ermakova, E. A., Shnol, E. E., Panasenko, G. P., and Ataullakhanov, F. I. (2011b). Finite platelet size could be responsible for platelet margination effect. Biophys. J. 101, 1835-1843. doi:10.1016/j.bpj.2011. 08.031

Ward, M. D., and Hammer, D. A. (1993). A theoretical analysis for the effect of focal contact formation on cell-substrate attachment strength. Biophys. J. 64, 936-959. doi:10.1016/S0006-3495(93)81456-5

Xu, Z. L., Chen, N., Shadden, S. C., Marsden, J. E., Kamocka, M. M., Rosen, E. D., et al. (2009). Study of blood flow impact on growth of thrombi using a multiscale model. Soft Matter 5, 769-779. doi:10.1039/b812429a

Zhao, R., Marhefka, J. N., Shu, F., Hund, S. J., Kameneva, M. V., and Antaki, J. F. (2008). Micro-flow visualization of red blood cell-enhanced platelet concentration at sudden expansion. Ann. Biomed. Eng. 36, 1130-1141. doi:10.1007/s10439008-9494-z

Conflict of Interest Statement: The authors declare that the research was conducted in the absence of any commercial or financial relationships that could be construed as a potential conflict of interest.

Received: 13 August 2013; paper pending published: 17 October 2013; accepted: 20 November 2013; published online: 03 December 2013.

Citation: Chesnutt JKW and Han H-C (2013) Effect of red blood cells on platelet activation and thrombus formation in tortuous arterioles. Front. Bioeng. Biotechnol. 1:18 doi: 10.3389/fbioe.2013.00018

This article was submitted to Biomechanics, a section of the journal Frontiers in Bioengineering and Biotechnology.

Copyright (c) 2013 Chesnutt and Han. This is an open-access article distributed under the terms of the Creative Commons Attribution License (CC BY). The use, distribution or reproduction in other forums is permitted, provided the original author (s) or licensor are credited and that the original publication in this journal is cited, in accordance with accepted academic practice. No use, distribution or reproduction is permitted which does not comply with these terms. 